\title{
Oscillation of Second-Order Neutral Dynamic Equations with Mixed Arguments
}

\author{
Tao $\mathrm{Ji}^{1, *}$, Shuhong Tang ${ }^{1}$ and Ethiraju Thandapani ${ }^{2}$ \\ ${ }^{1}$ School of Information and Control Engineering, Weifang University, Weifang, Shandong 261061, P. R. China \\ ${ }^{2}$ Ramanujan Institute for Advanced Study in Mathematics, University of Madras, Chennai 600 005, India
}

Received: 19 Aug. 2013, Revised: 16 Nov. 2013, Accepted: 17 Nov. 2013

Published online: 1 Sep. 2014

\begin{abstract}
We present some new oscillation criteria for a class of half-linear second-order neutral dynamic equations with mixed arguments. An example is given to illustrate the main results.
\end{abstract}

Keywords: Oscillation, neutral dynamic equation with mixed arguments, time scale

\section{Introduction}

This paper is concerned with oscillation of a second-order half-linear neutral functional dynamic equation

$$
\begin{aligned}
& \left(r(t)\left(\left(x(t)+p_{1}(t) x\left(\eta_{1}(t)\right)+p_{2}(t) x\left(\eta_{2}(t)\right)\right)^{\Delta}\right)^{\gamma}\right)^{\Delta} \\
& +q_{1}(t) x^{\gamma}\left(\tau_{1}(t)\right)+q_{2}(t) x^{\gamma}\left(\tau_{2}(t)\right)=0
\end{aligned}
$$

on an arbitrary time scale $\mathbb{T}$ unbounded above, where $\gamma$ is a quotient of odd positive integers, $r, p_{1}, p_{2}, q_{1}$, and $q_{2}$ are real-valued positive rd-continuous functions on $\mathbb{T}$. Also, we assume that $\eta_{1}, \eta_{2}, \tau_{1}, \tau_{2}: \mathbb{T} \rightarrow \mathbb{T}$ are rd-continuous, $\eta_{1}(t) \leq t, \eta_{2}(t) \geq t, \tau_{1}(t) \leq t, \tau_{2}(t) \geq t$, and $\lim _{t \rightarrow \infty} \eta_{1}(t)=\lim _{t \rightarrow \infty} \tau_{1}(t)=\infty$. Since we are interested in oscillatory behavior, we assume $t_{0} \in \mathbb{T}$ and it is convenient to assume $t_{0}>0$, and define the time scale interval of the form $\left[t_{0}, \infty\right)_{\mathbb{T}}$ by $\left[t_{0}, \infty\right)_{\mathbb{T}}:=\left[t_{0}, \infty\right) \cap \mathbb{T}$.

Set $z(t):=x(t)+p_{1}(t) x\left(\eta_{1}(t)\right)+p_{2}(t) x\left(\eta_{2}(t)\right)$. By a solution of (1) we mean a non-trivial real-valued function $x \in \mathrm{C}_{\mathrm{rd}}^{1}\left[T_{x}, \infty\right)_{\mathbb{T}}, T_{x} \in\left[t_{0}, \infty\right)_{\mathbb{T}}$ which has the properties that $z$ and $r\left(z^{\Delta}\right)^{\gamma}$ are defined and $\Delta$-differentiable for $t \in \mathbb{T}$, and satisfies (1) for $t \in\left[T_{x}, \infty\right)_{\mathbb{T}}$. The solutions vanishing in some neighbourhood of infinity will be excluded from our consideration. A solution $x$ of (1) is said to be oscillatory if it is neither eventually positive nor eventually negative; otherwise, it is termed nonoscillatory. Equation (1) is called oscillatory if all its solutions are oscillatory.

The study of dynamic equations on time scales, which goes back to its founder Hilger [1], is an area of mathematics that has recently received a lot of attention. Several authors have expounded on various aspects of this new theory, see the survey paper by Agarwal et al. [2] and the references cited therein. The books on the subject of time scales, i.e., measure chain, by Bohner and Peterson $[3,4]$, summarize and organize much of time scale calculus. In recent years, there has been much research activity concerning the oscillation and nonoscillation of solutions to different classes of dynamic equations on time scales, we refer the reader to the papers [5-25] and the references cited therein. Therein, Agarwal et al. [6], Candan [7], Erbe et al. [8], Şahiner [10], Saker [11], Saker et al. [12,13], Saker and O'Regan [14], Tripathy [15], Chen [16], Zhang and Wang [17], Wu et al. [18], and Thandapani et al. [20] studied a class of half-linear dynamic equations

$$
\left(r(t)\left(\left(x(t)+p_{1}(t) x\left(\eta_{1}(t)\right)\right)^{\Delta}\right)^{\gamma}\right)^{\Delta}+q_{1}(t) x^{\gamma}\left(\tau_{1}(t)\right)=0,
$$

where $\eta_{1}(t) \leq t$, and obtained some oscillation results under the assumption that

$$
\int_{t_{0}}^{\infty} \frac{\Delta t}{r^{1 / \gamma(t)}}=\infty
$$

The purpose of this paper is to derive new oscillation criteria for (1) in the cases where (2) holds or

$$
\int_{t_{0}}^{\infty} \frac{\Delta t}{r^{1 / \gamma}(t)}<\infty
$$

\footnotetext{
*Corresponding author e-mail: wfxytang@ 163.com
} 
In what follows, all functional inequalities are assumed to hold eventually, that is, they are satisfied for all $t$ large enough.

\section{Main results}

In this section, using the Riccati transformation technique, we establish some oscillation criteria for (1). In the sequel, we let

$d_{+}(t):=\max \{0, d(t)\}, \quad$ and $\quad d_{-}(t):=\max \{0,-d(t)\}$.

Theorem 2.1. Let (2) hold. Assume that there exists a positive real-valued $\Delta$-differentiable function $\delta$ such that for all sufficiently large $t_{1}$ and for $\tau_{1}(T)>t_{1}$,

$$
\limsup _{t \rightarrow \infty} \int_{T}^{t}\left[\delta(s) f(s)-\frac{r(s)\left(\delta_{+}^{\Delta}(s)\right)^{\gamma+1}}{(\gamma+1)^{\gamma+1} \delta^{\gamma}(s)}\right] \Delta s=\infty,
$$

where

$$
\begin{aligned}
f(s):= & q_{1}(s)\left(\frac{m\left(\tau_{1}(s)\right)}{m(s)}\right)^{\gamma} \\
& \cdot\left(1-p_{1}\left(\tau_{1}(s)\right)-p_{2}\left(\tau_{1}(s)\right) \frac{m\left(\eta_{2}\left(\tau_{1}(s)\right)\right)}{m\left(\tau_{1}(s)\right)}\right)^{\gamma} \\
& +q_{2}(s) \\
& \cdot\left(1-p_{1}\left(\tau_{2}(s)\right)-p_{2}\left(\tau_{2}(s)\right) \frac{m\left(\eta_{2}\left(\tau_{2}(s)\right)\right)}{m\left(\tau_{2}(s)\right)}\right)^{\gamma}
\end{aligned}
$$

and $m$ is a positive real-valued $\Delta$-differentiable function such that

$$
\frac{m(t)}{r^{1 / \gamma}(t) \int_{t_{1}}^{t} \frac{1}{r^{1 / \gamma(s)}} \Delta s}-m^{\Delta}(t) \leq 0
$$

and

$$
1-p_{1}(t)-p_{2}(t) \frac{m\left(\eta_{2}(t)\right)}{m(t)}>0 .
$$

Then (1) is oscillatory.

Proof. Let $x$ be a nonoscillatory solution of (1). Without loss of generality, we assume $x(t)>0, x\left(\eta_{i}(t)\right)>0$, and $x\left(\tau_{i}(t)\right)>0$ for $i=1,2$ and $t \in\left[t_{0}, \infty\right)_{\mathbb{T}}$. In view of (1), we obtain

$$
\begin{aligned}
\left(r(t)\left(z^{\Delta}(t)\right)^{\gamma}\right)^{\Delta}= & -q_{1}(t) x^{\gamma}\left(\tau_{1}(t)\right) \\
& -q_{2}(t) x^{\gamma}\left(\tau_{2}(t)\right)<0, \quad t \in\left[t_{0}, \infty\right)_{\mathbb{T}} .
\end{aligned}
$$

It follows from (2) that there exists a $t_{1} \in\left[t_{0}, \infty\right)_{\mathbb{T}}$ such that $z^{\Delta}(t)>0$ for $t \in\left[t_{1}, \infty\right)_{\mathbb{T}}$. By virtue of (8), we see that

$$
\begin{aligned}
z(t) & =z\left(t_{1}\right)+\int_{t_{1}}^{t} \frac{\left(r(s)\left(z^{\Delta}(s)\right)^{\gamma}\right)^{1 / \gamma}}{r^{1 / \gamma}(s)} \Delta s \\
& \geq r^{1 / \gamma}(t) z^{\Delta}(t) \int_{t_{1}}^{t} \frac{\Delta s}{r^{1 / \gamma}(s)} .
\end{aligned}
$$

Since

$$
\begin{aligned}
\left(\frac{z(t)}{m(t)}\right)^{\Delta} & =\frac{z^{\Delta}(t) m(t)-z(t) m^{\Delta}(t)}{m(t) m^{\sigma}(t)} \\
& \leq \frac{z(t)}{m(t) m^{\sigma}(t)}\left[\frac{m(t)}{r^{1 / \gamma}(t) \int_{t_{1}}^{t} \frac{1}{r^{1 / \gamma}(s)} \Delta s}-m^{\Delta}(t)\right] \leq 0,
\end{aligned}
$$

we find that $z / m$ is nonincreasing. Hence we have

$$
\begin{aligned}
x(t) & =z(t)-p_{1}(t) x\left(\eta_{1}(t)\right)-p_{2}(t) x\left(\eta_{2}(t)\right) \\
& \geq z(t)-p_{1}(t) z\left(\eta_{1}(t)\right)-p_{2}(t) z\left(\eta_{2}(t)\right) \\
& \geq\left(1-p_{1}(t)-p_{2}(t) \frac{m\left(\eta_{2}(t)\right)}{m(t)}\right) z(t) .
\end{aligned}
$$

By virtue of (8), we have

$$
\begin{aligned}
& \left(r(t)\left(z^{\Delta}(t)\right)^{\gamma}\right)^{\Delta} \leq-q_{1}(t) \\
& \left(1-p_{1}\left(\tau_{1}(t)\right)-p_{2}\left(\tau_{1}(t)\right) \frac{m\left(\eta_{2}\left(\tau_{1}(t)\right)\right)}{m\left(\tau_{1}(t)\right)}\right)^{\gamma} z^{\gamma}\left(\tau_{1}(t)\right) \\
- & q_{2}(t)\left(1-p_{1}\left(\tau_{2}(t)\right)-p_{2}\left(\tau_{2}(t)\right) \frac{m\left(\eta_{2}\left(\tau_{2}(t)\right)\right)}{m\left(\tau_{2}(t)\right)}\right)^{\gamma} \\
& \cdot z^{\gamma}\left(\tau_{2}(t)\right) .
\end{aligned}
$$

Define the function $\omega$ by

$$
\omega(t):=\delta(t) \frac{r(t)\left(z^{\Delta}(t)\right)^{\gamma}}{z^{\gamma}(t)}, \quad t \in\left[t_{1}, \infty\right)_{\mathbb{T}} .
$$

Then $\omega(t)>0$ for $t \in\left[t_{1}, \infty\right)_{\mathbb{T}}$. The rest of the proof is similar to that of [8, Theorem 2.1], and so is omitted. The proof is complete.

Remark 2.1. The function $m$ is existent, see, e.g., $m(t)=$ $\int_{t_{1}}^{t} \frac{1}{r^{1 / \gamma}(s)} \Delta s$.

Theorem 2.2. Let (2) hold. Assume that there exist functions $H, h$ such that for each fixed $t, H(t, s)$ and $h(t, s)$ are rd-continuous with respect to $s$ on $\mathbb{D}=\left\{(t, s): t \geq s \geq t_{0}\right\}$ and

$$
H(t, t)=0, \quad t \geq t_{0}, \quad H(t, s)>0, \quad t>s \geq t_{0},
$$

and $H$ has a nonpositive continuous $\Delta$-partial derivative $H^{\Delta_{s}}(t, s)$ with respect to the second variable and satisfies

$$
\begin{array}{r}
-H^{\Delta_{s}}(t, s)-H(t, s) \frac{\delta^{\Delta}(s)}{\delta^{\sigma}(s)} \\
=\frac{h(t, s)}{\delta^{\sigma}(s)}(H(t, s))^{\gamma /(\gamma+1)},
\end{array}
$$

and for all sufficiently large $t_{1}$ and for $\tau_{1}(T)>t_{1}$,

$$
\begin{aligned}
\limsup _{t \rightarrow \infty} \frac{1}{H(t, T)} \int_{T}^{t} & {[\delta(s) H(t, s) f(s)} \\
& \left.-\frac{r(s)\left(h_{-}(t, s)\right)^{\gamma+1}}{(\gamma+1)^{\gamma+1} \delta^{\gamma}(s)}\right] \Delta s=\infty,
\end{aligned}
$$

where $f$ is defined as in (5), $m$ is a positive real-valued $\Delta$ differentiable function such that (6) and (7) hold, and $\delta$ is 
a positive real-valued $\Delta$-differentiable function. Then (1) is oscillatory.

Proof. From Theorem 2.1, the proof is similar to that of [8, Theorem 2.2], and hence is omitted. This completes the proof.

Theorem 2.3. Let (3) hold and $0 \leq p_{1}(t)+p_{2}(t) \leq p<1$. Assume that all assumptions of Theorem 2.1 or Theorem 2.2 hold except (2). If for all sufficiently large $t_{1}$,

$$
\int_{t_{1}}^{\infty}\left[\frac{1}{r(s)} \int_{t_{1}}^{s}\left[q_{1}(\tau)+q_{2}(\tau)\right] \Delta \tau\right]^{1 / \gamma} \Delta s=\infty
$$

then every solution of (1) is oscillatory or converges to zero as $t \rightarrow \infty$.

Proof. Let $x$ be a nonoscillatory solution of (1). Without loss of generality, we assume $x(t)>0, x\left(\eta_{i}(t)\right)>0$, and $x\left(\tau_{i}(t)\right)>0$ for $i=1,2$ and $t \in\left[t_{0}, \infty\right)_{\mathbb{T}}$. In view of (1), we obtain (8). Then there exists a $t_{1} \in\left[t_{0}, \infty\right)_{\mathbb{T}}$ such that $z^{\Delta}(t)>0$, or $z^{\Delta}(t)<0$ for $t \in\left[t_{1}, \infty\right)_{\mathbb{T}}$.

Assume first that $z^{\Delta}(t)>0$ for $t \in\left[t_{1}, \infty\right)_{\mathbb{T}}$. As in Theorem 2.1 or Theorem 2.2, we can obtain the corresponding contradictions. Assume now that $z^{\Delta}(t)<0$ for $t \in\left[t_{1}, \infty\right)_{\mathbb{T}}$. We assert that $\lim _{t \rightarrow \infty} z(t)=0$. If not, similar to the proof of [26, Lemma 2], there exist two constants $k>0$ and $l>0$ such that

$$
x(t) \geq k z(t) \geq k l .
$$

By virtue of (8), we have

$$
\begin{aligned}
\left(r(t)\left(z^{\Delta}(t)\right)^{\gamma}\right)^{\Delta} & =-q_{1}(t) x^{\gamma}\left(\tau_{1}(t)\right)-q_{2}(t) x^{\gamma}\left(\tau_{2}(t)\right) \\
& \leq-(k l)^{\gamma}\left[q_{1}(t)+q_{2}(t)\right] .
\end{aligned}
$$

Integrating the latter inequality from $t_{1}$ to $t$, we get

$$
r(t)\left(z^{\Delta}(t)\right)^{\gamma} \leq-(k l)^{\gamma} \int_{t_{1}}^{t}\left[q_{1}(s)+q_{2}(s)\right] \Delta s .
$$

Integrating again from $t_{1}$ to $t$, we obtain

$$
z(t) \leq z\left(t_{1}\right)-k l \int_{t_{1}}^{t}\left[\frac{1}{r(s)} \int_{t_{1}}^{s}\left[q_{1}(\tau)+q_{2}(\tau)\right] \Delta \tau\right]^{1 / \gamma} \Delta s
$$

Hence by (14), $\lim _{t \rightarrow \infty} z(t)=-\infty$, which is a contradiction. Therefore, $\lim _{t \rightarrow \infty} z(t)=0$. Then, $\lim _{t \rightarrow \infty} x(t)=0$ due to $0 \leq x(t) \leq z(t)$. This completes the proof.

For an application of our results, we give the following example.

Example 2.1. We consider the second-order neutral functional dynamic equation

$$
\begin{aligned}
& \left(x(t)+\frac{1}{2} x\left(\eta_{1}(t)\right)+\frac{t^{2}}{3\left(\eta_{2}(t)\right)^{2}} x\left(\eta_{2}(t)\right)\right)^{\Delta \Delta} \\
& +\frac{k_{1}}{t} x\left(\tau_{1}(t)\right)+\frac{k_{2}}{t} x\left(\tau_{2}(t)\right)=0
\end{aligned}
$$

where $t \in[1, \infty)_{\mathbb{T}}, \tau_{1}(t) \leq t, \tau_{2}(t) \geq t, \eta_{1}(t) \leq t, \eta_{2}(t) \geq t$, $k_{1}>0$, and $k_{2}>0$. Let $m(t)=t^{2}$ and $\delta(t)=1$. Then, every solution of (15) is oscillatory when using Theorem 2.1.

\section{Acknowledgement}

This research is supported by Natural Science Foundation of Shandong Province (Z2007F08) and also by Weifang University Research Funds for Doctors (2012BS25).

\section{References}

[1] S. Hilger. Analysis on measure chains-a unified approach to continuous and discrete calculus. Results Math., 18, 18-56 (1990).

[2] R. P. Agarwal, M. Bohner, D. O'Regan, A. Peterson. Dynamic equations on time scales: a survey. J. Comput. Appl. Math., 141, 1-26 (2002).

[3] M. Bohner, A. Peterson. Dynamic Equations on Time Scales: An Introduction with Applications. Birkhäuser, Boston, (2001).

[4] M. Bohner, A. Peterson. Advances in Dynamic Equations on Time Scales. Birkhäuser, Boston, (2003).

[5] R. P. Agarwal, S. R. Grace, D. O'Regan. Oscillation Theory for Second Order Linear, Half-Linear, Superlinear and Sublinear Dynamic Equations. Kluwer Academic Publishers, Dordrecht, (2002).

[6] R. P. Agarwal, D. O'Regan, S. H. Saker. Oscillation criteria for second-order nonlinear neutral delay dynamic equations. J. Math. Anal. Appl., 300, 203-217 (2004).

[7] T. Candan. Oscillation criteria for second-order nonlinear neutral dynamic equations with distributed deviating arguments on time scales. Adv. Difference Eq., 2013, 1-8 (2013).

[8] L. Erbe, T. S. Hassan, A. Peterson. Oscillation criteria for nonlinear functional neutral dynamic equations on time scales. J. Difference Equ. Appl., 15, 1097-1116 (2009).

[9] M. T. Şenel. Kamenev-type oscillation criteria for the secondorder nonlinear dynamic equations with damping on time scales. Abstr. Appl. Anal., 2012, 1-18 (2012).

[10] Y. Şahiner. Oscillation of second order neutral delay and mixed type dynamic equations on time scales. Adv. Difference Eq., 2006, 1-9 (2006).

[11] S. H. Saker. Oscillation of second-order nonlinear neutral delay dynamic equations on time scales. J. Comput. Appl. Math., 187, 123-141 (2006).

[12] S. H. Saker, R. P. Agarwal, D. O'Regan. Oscillation theorems for second-order nonlinear neutral delay dynamic equations on time scales. Acta Math. Sci., 24, 1409-1432 (2008).

[13] S. H. Saker, R. P. Agarwal, D. O'Regan. Oscillation results for second-order nonlinear neutral delay dynamic equations on time scales. Applicable Anal., 86, 1-17 (2007).

[14] S. H. Saker, D. O'Regan. New oscillation criteria for second-order neutral functional dynamic equations via the generalized Riccati substitution. Commun. Nonlinear Sci. Numer. Simulat., 16, 423-434 (2011).

[15] A. K. Tripathy. Some oscillation results for second order nonlinear dynamic equations of neutral type. Nonlinear Anal., 71, e1727-e1735 (2009).

[16] D. X. Chen. Oscillation of second-order Emden-Fowler neutral delay dynamic equations on time scales. Math. Comput. Modelling, 51, 1221-1229 (2010). 
[17] S. Y. Zhang, Q. R. Wang. Oscillation of second-order nonlinear neutral dynamic equations on time scales. Appl. Math. Comput., 216, 2837-2848 (2010).

[18] H. W. Wu, R. K. Zhuang, R. M. Mathsen. Oscillation criteria for second-order nonlinear neutral variable delay dynamic equations. Appl. Math. Comput., 178, 321-331 (2006).

[19] B. Karpuz. Asymptotic behavior of bounded solutions of a class of higher-order neutral dynamic equations. Appl. Math. Comput., 215, 2174-2183 (2009).

[20] E. Thandapani, V. Piramanantham, S. Pinelas. Oscillation criteria for second order neutral delay dynamic equations with mixed nonlinearities. Adv. Difference Eq., 2011, 1-14 (2011).

[21] Z. Han, T. Li, S. Sun, C. Zhang. On the oscillation of secondorder neutral delay dynamic equations on time scales. Afri. Dias. J. Math., 9, 76-86 (2010).

[22] Z. Han, T. Li, S. Sun, C. Zhang, B. Han. Oscillation criteria for a class of second-order neutral delay dynamic equations of Emden-Fowler type. Abstr. Appl. Anal., 2011, 1-26 (2011).

[23] E. Thandapani, V. Piramanantham. Oscillation criteria of second order neutral delay dynamic equations with distributed deviating arguments. Electron. J. Qual. Theory Differ. Equ., 61, 1-15 (2010).

[24] T. Li, Z. Han, S. Sun, D. Yang. Existence of nonoscillatory solutions to second-order neutral delay dynamic equations on time scales. Adv. Difference Eq., 2009, 1-10 (2009).

[25] T. Li. Comparison theorems for second-order neutral differential equations of mixed type. Electron. J. Diff. Equ., 167, 1-7 (2010).

[26] B. Baculíková, J. Džurina. Oscillation of third-order neutral differential equations. Math. Comput. Modelling, 52, 215226 (2010).

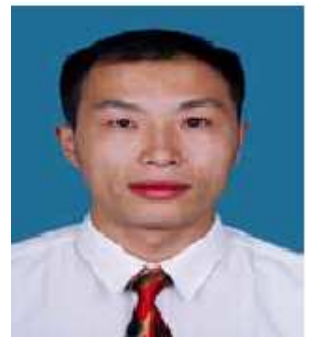

Tao Ji received the Ph.D. in control theory and control engineering from the Shandong University. He is now an associate professor of Department of Information and Automatic Engineering in Weifang University. He has published and authored more than 30 papers on academic journals and conference. His main research interests include: optimal control technique, heating energy-saving new technology and applications, and urban public building energy-saving system solutions.

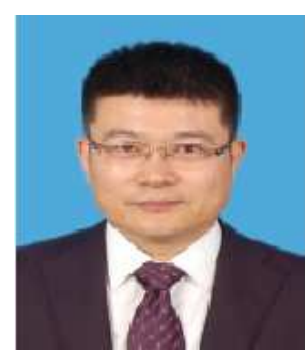

Shuhong

Tang received the Ph.D. in ocean information exploring and processing from the Ocean University of China. He is now a professor of Department of Information and Automatic Engineering in Weifang University. His main research interests include: intelligent control and qualitative analysis of dynamic equations.

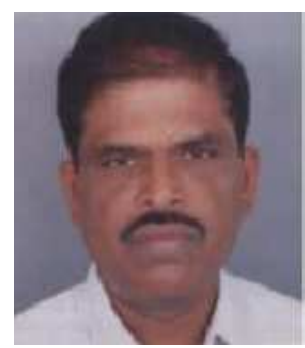

\section{Ethiraju Thandapani} received the Ph.D. in Mathematical Sciences from the University of Madras. $\mathrm{He}$ is now a professor of Ramanujan Institute for Advanced Study in Mathematics in University of Madras. His main research interests include: general area of research is ordinary and functional differential equations, difference equations, and their applications. Special interests include: nonlinear oscillations (stability, oscillation, nonoscillation, and other asymptotic properties of solutions of nonlinear equations), oscillation theory of partial functional differential equations, differential, difference equations and integral inequalities, numerical solution of ordinary and partial differential equations. 